\title{
ON THE CROSSING NUMBER OF THE JOIN OF FIVE VERTEX GRAPH G WITH THE DISCRETE GRAPH $D_{n}$
}

\author{
Štefan BEREŽNÝ, Michal STAŠ \\ Department of Mathematics and Theoretical Informatic, Faculty of Electrical Engineering and Informatics, Technical University of \\ Košice, Boženy Němcovej 32, 04200 Košice, Slovak Republic, Tel.: +421 55602 2447, \\ E-mail: stefan.berezny@tuke.sk, michal.stas@tuke.sk
}

\begin{abstract}
In this paper, we show the values of crossing numbers for join products of graph $G$ on five vertices with the discrete graph $D_{n}$ and the path $P_{n}$ on $n$ vertices. The proof is done with the help of software. The software generates all cyclic permutations for a given number $n$. For cyclic permutations, $P_{1}-P_{m}$ will create a graph in which to calculate the distances between all vertices of the graph. These distances are used in proof of crossing numbers of presented graphs.
\end{abstract}

Keywords: crossing number, cyclic permutations, drawing, graph, join

\section{INTRODUCTION}

Let $G$ be a simple graph with the vertex set $V$ and the edge set $E$. A drawing of the graph $G$ is a representation of $G$ in the plane such that its vertices are represented by distinct points and its edges by simple continuous arcs connecting the corresponding point pairs. In such a drawing, the intersection of the interiors of the arcs is called a crossing. We assume that in a drawing no edge passes through any vertex other than its end-points, no two edges touch each other (i.e., if two edges have a common interior point, then they cross properly at this point), and no three edges cross at the same point. It is easy to see that a drawing with minimum number of crossings (an optimal drawing) is always a good drawing, meaning that no edge crosses itself, no two edges cross more than once, and no two edges incident with the same vertex cross each other.

The crossing number $\operatorname{cr}(G)$ of a simple graph $G$ with the vertex set $V(G)$ and the edge set $E(G)$ is defined as the minimum possible number of edge crossings in a drawing of $G$ in the plane.

Let $G_{1}=\left(V\left(G_{1}\right), E\left(G_{1}\right)\right)$ and $G_{2}=\left(V\left(G_{2}\right), E\left(G_{2}\right)\right)$ be simple graphs. The join product of two graphs $G_{1}$ and $G_{2}$, denoted by $G_{1}+G_{2}$, is obtained from the vertex-disjoint copies of $G_{1}$ and $G_{2}$ by adding all edges between $V\left(G_{1}\right)$ and $V\left(G_{2}\right)$. For $\left|V\left(G_{1}\right)\right|=m$, and $\left|V\left(G_{2}\right)\right|=n$, the edge set of $G_{1}+G_{2}$ is the union of disjoint edge sets of the graphs $G_{1}, G_{2}$, and the complete bipartite graph $K_{m, n}$.

Let $D(D(G))$ be a good drawing of the graph $G$. We denote the number of crossings in $D$ by $\operatorname{cr}_{D}(G)$. Let $G_{i}$ and $G_{j}$ be edge-disjoint subgraphs of $G$. We denote the number of crossings between edges of $G_{i}$ and edges of $G_{j}$ by $\operatorname{cr}_{D}\left(G_{i}, G_{j}\right)$, and the number of crossings among edges of $G_{i}$ in $D$ by $\operatorname{cr}_{D}\left(G_{i}\right)$. It is easy to see that for any three mutually edge-disjoint subgraphs $G_{i}, G_{j}$, and $G_{k}$ of $G$, the following equations hold:

$\operatorname{cr}_{D}\left(G_{i} \cup G_{j}\right)=\operatorname{cr}_{D}\left(G_{i}\right)+\operatorname{cr}_{D}\left(G_{j}\right)+\operatorname{cr}_{D}\left(G_{i}, G_{j}\right)$,

$\operatorname{cr}_{D}\left(G_{i} \cup G_{j}, G_{k}\right)=\operatorname{cr}_{D}\left(G_{i}, G_{k}\right)+\operatorname{cr}_{D}\left(G_{j}, G_{k}\right)$.

In the paper, some proofs are based on the Kleitman's result on crossing numbers of complete bipartite graphs. More precisely, he proved that

$\operatorname{cr}\left(K_{m, n}\right)=\left\lfloor\frac{m}{2}\right\rfloor\left\lfloor\frac{m-1}{2}\right\rfloor\left\lfloor\frac{n}{2}\right\rfloor\left\lfloor\frac{n-1}{2}\right\rfloor, \quad$ if $\quad m \leq 6$.

\section{SUBJECT}

\subsection{Software description}

We will describe in this subchapter the software which we use when proving the Theorem 4.1 in this article and also in a similar proofs of theorems such like this. The input for the algorithm is the number $n$, which represents an $n$-element set $\{1,2,3, \ldots, n\}$. The algorithm selects all cyclic permutations from the set of all permutations of the $n$-element set $\{1,2,3, \ldots, n\}$. The software marks these permutations with symbols $P_{1}, \ldots, P_{m}$, where $m=(n-1)$ !. Said software gives outputs of distance between each pair of vertices of given graph.

A graph is created with a set of vertices $V=$ $\left\{P_{1}, P_{2}, \ldots, P_{m}\right\}$ and set of edges $E$, where the two vertices are joined by the edge if the vertices correspond to the permutations $P_{i}$ and $P_{j}$, which are formed by the exchange of exactly two elements of the $n$-tuple (i. e. an ordered set with $n$ elements). This graph is represented by a square symmetrical adjacency matrix. The distance between each pair of vertices are calculated using the properties of the cyclicorder graph $\mathrm{CO}_{5}$ defined in [5].

The software uses the following graph theory:

Let us denote $B^{(1)}$ the matrix is gotten from adjacency matrix $B$ by adding ones to the main diagonal. Let us consider the matrix $B^{(2)}=\left\{b_{i j}^{(2)}\right\}_{i, j=1}^{m}$ such that $B^{(2)}=B^{(1)} \cdot B^{(1)}$. From the matrix multiplication it is obvious that $b_{i j}^{(2)}=$ $\sum_{k=1}^{m} b_{i k}^{(1)} \cdot b_{k j}^{(1)}$, but in this matrix we will use the Boolean addition and multiplication $(1 \cdot 1=1,0 \cdot 1=1 \cdot 0=0 \cdot 0=0$, $1+0=0+1=1+1=1$, and $0+0=0)$. Generally we can consider matrix $B^{(m)}=B^{(m-1)} \cdot B^{(1)}$.

Theorem 2.1. Let the adjacency matrix $B$ of the connected graph $G=(V, H),|V|=n$ is given. Then for arbitrary $k=1,2, \ldots, m$, the element $b_{i j}^{(k)}$ of the matrix $B^{(k)}$ is equal to one if $d\left(v_{i}, v_{j}\right) \leq k$.

Corollary 2.1. The graph $G=(V, H),|V|=n$, is con- 
nected only when the elements of the matrix $B^{(n-1)}$ are only ones.

Corollary 2.2. For each two different vertices of the graph $G=(V, H), d\left(v_{i}, v_{j}\right)=\min _{k \in\{1,2, \ldots, m\}}\left\{k ; b_{i j}^{(k)}=1\right\}$.

\subsection{Important facts}

We will show the correct proof of the theorem from the article [1]. We deal with the graph $G$ with the vertex set $V=\left\{v_{1}, v_{2}, v_{3}, v_{4}, v_{5}\right\}$ which is shown in Fig. 11. There is also the graph $G$ with renamed vertices $V=\{1,2,3,4,5\}$, which is done counter-clockwise with the beginning in the upper right corner.
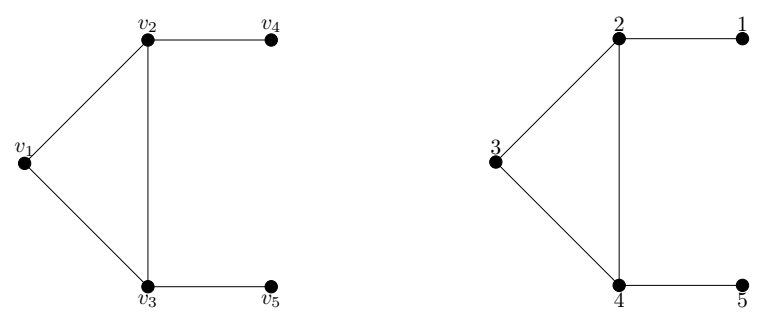

Fig. 1 Five vertex graph $G$ and its numbering of vertices

Using the given software for $n=5$, we get the names of the cyclic permutations (see Table 1) and the distance between the cyclic permutations (see Table 22- 5). The maximum distance between two vertices in the graph is equal to four. With this information we can use arguments which are described in [3, 4].

Table 1 Names of Cyclic Permutations of 5-elements set

\begin{tabular}{||ll||ll||}
\hline \hline Name & Cyclic perm. & Name & Cyclic perm. \\
\hline \hline$P_{1} \longrightarrow$ & $(12345)$ & $P_{13} \longrightarrow$ & $(12543)$ \\
$P_{2} \longrightarrow$ & $(13245)$ & $P_{14} \longrightarrow$ & $(15243)$ \\
$P_{3} \longrightarrow$ & $(12435)$ & $P_{15} \longrightarrow$ & $(12453)$ \\
$P_{4} \longrightarrow$ & $(14235)$ & $P_{16} \longrightarrow$ & $(14253)$ \\
$P_{5} \longrightarrow$ & $(14325)$ & $P_{17} \longrightarrow$ & $(14523)$ \\
$P_{6} \longrightarrow$ & $(13425)$ & $P_{18} \longrightarrow$ & $(15423)$ \\
$P_{7} \longrightarrow$ & $(12354)$ & $P_{19} \longrightarrow$ & $(15342)$ \\
$P_{8} \longrightarrow$ & $(13254)$ & $P_{20} \longrightarrow$ & $(13542)$ \\
$P_{9} \longrightarrow$ & $(12534)$ & $P_{21} \longrightarrow$ & $(15432)$ \\
$P_{10} \longrightarrow$ & $(15234)$ & $P_{22} \longrightarrow$ & $(14532)$ \\
$P_{11} \longrightarrow$ & $(15324)$ & $P_{23} \longrightarrow$ & $(14352)$ \\
$P_{12} \longrightarrow$ & $(13524)$ & $P_{24} \longrightarrow$ & $(13452)$ \\
\hline \hline
\end{tabular}

\section{METHODS}

We consider the join of $G$ with the discrete graph on $n$ vertices $D_{n}$. The graph $G+D_{n}$ consists of one copy of the graph $G$ and of $n$ vertices $t_{1}, t_{2}, \ldots, t_{n}$, where any vertex $t_{i}, i=1,2, \ldots, n$, is adjacent to every vertex of $G$. Let $T^{i}$, $1 \leq i \leq n$, denote the subgraph induced by the five edges incident with the vertex $t_{i}$. Then

$$
G+D_{n}=G \cup K_{5, n}=G \cup\left(\bigcup_{i=1}^{n} T^{i}\right) .
$$

The graph $G+D_{1}$ is planar, thus $\operatorname{cr}\left(G+D_{1}\right)=0$. One can easy to verify that $\operatorname{cr}\left(G+D_{2}\right) \leq 1$. The graph $G+D_{2}$ contains a subdivision of $K_{3,3}$ as a subgraph, and therefore $\operatorname{cr}\left(G+D_{2}\right) \geq 1$. So, $\operatorname{cr}\left(G+D_{2}\right)=1$.

Let $D$ be a good drawing of the graph $G+D_{n}$. The rotation $\operatorname{rot}_{D}\left(t_{i}\right)$ of vertex a $t_{i}$ in the drawing $D$ is the cyclic permutation that records the (cyclic) counter-clockwise order in which the edges leave $t_{i}$, see [4]. We use the notation (12543) if the counter-clockwise order the edges incident with the vertex $t_{i}$ is $t_{i} 1, t_{i} 2, t_{i} 5, t_{i} 4$, and $t_{i} 3$ (see $A_{1}$ in Fig. 2), where $V=\{1,2,3,4,5\}$ are noted vertices of the graph $G$. We emphasize that a rotation is a cyclic permutation. For $i, j \in\{1,2, \ldots, n\}, i \neq j$, every subgraph $T^{i} \cup T^{j}$ of the graph $G+D_{n}$ is isomorphic with the graph $K_{5,2}$. In the paper, we will deal with the minimum necessary number of crossings between the edges of $T^{i}$ and the edges of $T^{j}$ in a subgraph $T^{i} \cup T^{j}$ induced by the drawing $D$ of the graph $G+D_{n}$ depending on the rotations $\operatorname{rot}_{D}\left(t_{i}\right)$ and $\operatorname{rot}_{D}\left(t_{j}\right)$.

D. R. Woodall [5] proved that, in any good drawing $D$ of the graph $K_{5,2}, \operatorname{cr}_{D}\left(T^{i}, T^{j}\right) \geq 4$ if $\operatorname{rot}_{D}\left(t_{i}\right)=\operatorname{rot}_{D}\left(t_{j}\right)$. Moreover, if $Q\left(\operatorname{rot}_{D}\left(t_{i}\right), \operatorname{rot}_{D}\left(t_{j}\right)\right)$ denotes the minimum number of interchanges of adjacent elements of $\operatorname{rot}_{D}\left(t_{i}\right)$ required to produce the inverse cyclic permutation of $\operatorname{rot}_{D}\left(t_{j}\right)$, then $Q\left(\operatorname{rot}_{D}\left(t_{i}\right), \operatorname{rot}_{D}\left(t_{j}\right)\right) \leq \operatorname{cr}_{D}\left(T^{i}, T^{j}\right)$.

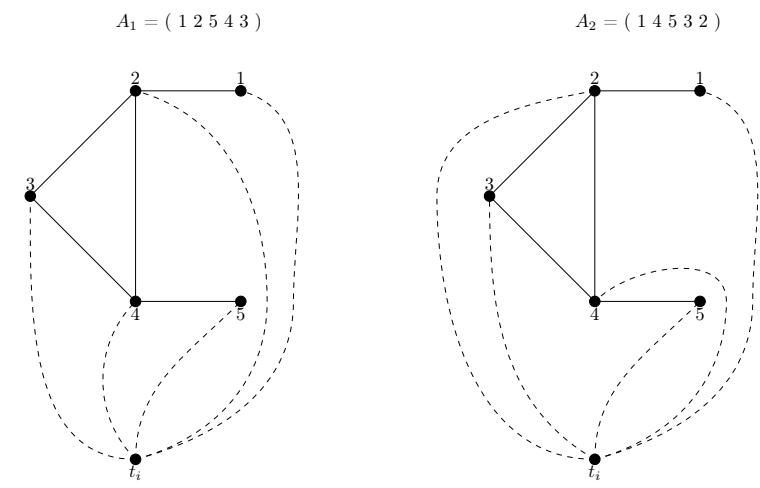

Fig. 2 Graph $G$ and its configurations - type $A$

We will separate the subgraphs $T^{i}, i=1, \ldots, n$, of $G+D_{n}$ into three subsets depending on how many the considered $T^{i}$ crosses the edges of $G$ in $D$. For $i=1,2, \ldots, n$, we denote by $R_{D}=\left\{T^{i}: \mathrm{cr}_{D}\left(G, T^{i}\right)=0\right\}$ and $S_{D}=\left\{T^{i}\right.$ : $\left.\operatorname{cr}_{D}\left(G, T^{i}\right)=1\right\}$. Every other subgraph $T^{i}$ crosses $G$ at least twice in $D$. Moreover, let $F^{i}$ denote the subgraph $G \cup T^{i}$ for $T^{i} \in R_{D}$, where $i \in\{1, \ldots, n\}$. Thus, any $F^{i}$ is exactly represented by $\operatorname{rot}_{D}\left(t_{i}\right)$. 
Table 2 Distance 1 Between Cyclic Permutations

\begin{tabular}{||ll||}
\hline \hline From & To \\
\hline \hline$P_{1}$ & $P_{2}, P_{3}, P_{7}, P_{10}, P_{24}$ \\
$P_{2}$ & $P_{1}, P_{6}, P_{8}, P_{11}, P_{15}$ \\
$P_{3}$ & $P_{1}, P_{4}, P_{14}, P_{15}, P_{23}$ \\
$P_{4}$ & $P_{3}, P_{5}, P_{7}, P_{16}, P_{18}$ \\
$P_{5}$ & $P_{4}, P_{6}, P_{8}, P_{21}, P_{23}$ \\
$P_{6}$ & $P_{2}, P_{5}, P_{16}, P_{19}, P_{24}$ \\
$P_{7}$ & $P_{1}, P_{4}, P_{8}, P_{9}, P_{20}$ \\
$P_{8}$ & $P_{2}, P_{5}, P_{7}, P_{12}, P_{13}$ \\
$P_{9}$ & $P_{7}, P_{10}, P_{13}, P_{16}, P_{19}$ \\
$P_{10}$ & $P_{1}, P_{9}, P_{11}, P_{14}, P_{17}$ \\
$P_{11}$ & $P_{2}, P_{10}, P_{12}, P_{19}, P_{22}$ \\
$P_{12}$ & $P_{8}, P_{11}, P_{14}, P_{20}, P_{23}$ \\
$P_{13}$ & $P_{8}, P_{9}, P_{14}, P_{15}, P_{21}$ \\
$P_{14}$ & $P_{3}, P_{10}, P_{12}, P_{13}, P_{18}$ \\
$P_{15}$ & $P_{2}, P_{3}, P_{13}, P_{16}, P_{22}$ \\
$P_{16}$ & $P_{4}, P_{6}, P_{9}, P_{15}, P_{17}$ \\
$P_{17}$ & $P_{10}, P_{16}, P_{18}, P_{22}, P_{24}$ \\
$P_{18}$ & $P_{4}, P_{14}, P_{17}, P_{20}, P_{21}$ \\
$P_{19}$ & $P_{6}, P_{9}, P_{11}, P_{20}, P_{21}$ \\
$P_{20}$ & $P_{7}, P_{12}, P_{18}, P_{19}, P_{24}$ \\
$P_{21}$ & $P_{5}, P_{13}, P_{18}, P_{19}, P_{22}$ \\
$P_{22}$ & $P_{11}, P_{15}, P_{17}, P_{21}, P_{23}$ \\
$P_{23}$ & $P_{3}, P_{5}, P_{12}, P_{22}, P_{24}$ \\
$P_{24}$ & $P_{1}, P_{6}, P_{17}, P_{20}, P_{23}$ \\
\hline & \\
\hline
\end{tabular}

Table 3 Distance 2 Between Cyclic Permutations

\begin{tabular}{|c|c|}
\hline From & To \\
\hline$P_{1}$ & $P_{4}, P_{6}, P_{8}, P_{9}, P_{11}, P_{14}, P_{15}, P_{17}, P_{20}, P_{23}$ \\
\hline$P_{2}$ & $P_{3}, P_{5}, P_{7}, P_{10}, P_{12}, P_{13}, P_{16}, P_{19}, P_{22}, P_{24}$ \\
\hline$P_{3}$ & $P_{2}, P_{5}, P_{7}, P_{10}, P_{12}, P_{13}, P_{16}, P_{18}, P_{22}, P_{24}$ \\
\hline$P_{4}$ & $P_{1}, P_{6}, P_{8}, P_{9}, P_{14}, P_{15}, P_{17}, P_{20}, P_{21}, P_{23}$ \\
\hline$P_{5}$ & $P_{2}, P_{3}, P_{7}, P_{12}, P_{13}, P_{16}, P_{18}, P_{19}, P_{22}, P_{24}$ \\
\hline$P_{6}$ & $P_{1}, P_{4}, P_{8}, P_{9}, P_{11}, P_{15}, P_{17}, P_{20}, P_{21}, P_{23}$ \\
\hline$P_{7}$ & $P_{2}, P_{3}, P_{5}, P_{10}, P_{12}, P_{13}, P_{16}, P_{18}, P_{19}, P_{24}$ \\
\hline$P_{8}$ & $P_{1}, P_{4}, P_{6}, P_{9}, P_{11}, P_{14}, P_{15}, P_{20}, P_{21}, P_{23}$ \\
\hline$P_{9}$ & $P_{1}, P_{4}, P_{6}, P_{8}, P_{11}, P_{14}, P_{15}, P_{17}, P_{20}, P_{21}$ \\
\hline$P_{10}$ & $P_{2}, P_{3}, P_{7}, P_{12}, P_{13}, P_{16}, P_{18}, P_{19}, P_{22}, P_{24}$ \\
\hline$P_{11}$ & $P_{1}, P_{6}, P_{8}, P_{9}, P_{14}, P_{15}, P_{17}, P_{20}, P_{21}, P_{23}$ \\
\hline$P_{12}$ & $P_{2}, P_{3}, P_{5}, P_{7}, P_{10}, P_{13}, P_{18}, P_{19}, P_{22}, P_{24}$ \\
\hline$P_{13}$ & $P_{2}, P_{3}, P_{5}, P_{7}, P_{10}, P_{12}, P_{16}, P_{18}, P_{19}, P_{22}$ \\
\hline$P_{14}$ & $P_{1}, P_{4}, P_{8}, P_{9}, P_{11}, P_{15}, P_{17}, P_{20}, P_{21}, P_{23}$ \\
\hline$P_{15}$ & $P_{1}, P_{4}, P_{6}, P_{8}, P_{9}, P_{11}, P_{14}, P_{17}, P_{21}, P_{23}$ \\
\hline$P_{16}$ & $P_{2}, P_{3}, P_{5}, P_{7}, P_{10}, P_{13}, P_{18}, P_{19}, P_{22}, P_{24}$ \\
\hline$P_{17}$ & $P_{1}, P_{4}, P_{6}, P_{9}, P_{11}, P_{14}, P_{15}, P_{20}, P_{21}, P_{23}$ \\
\hline$P_{18}$ & $P_{3}, P_{5}, P_{7}, P_{10}, P_{12}, P_{13}, P_{16}, P_{19}, P_{22}, P_{24}$ \\
\hline$P_{19}$ & $P_{2}, P_{5}, P_{7}, P_{10}, P_{12}, P_{13}, P_{16}, P_{18}, P_{22}, P_{24}$ \\
\hline$P_{20}$ & $P_{1}, P_{4}, P_{6}, P_{8}, P_{9}, P_{11}, P_{14}, P_{17}, P_{21}, P_{23}$ \\
\hline$P_{21}$ & $P_{4}, P_{6}, P_{8}, P_{9}, P_{11}, P_{14}, P_{15}, P_{17}, P_{20}, P_{23}$ \\
\hline$P_{22}$ & $P_{2}, P_{3}, P_{5}, P_{10}, P_{12}, P_{13}, P_{16}, P_{18}, P_{19}, P_{24}$ \\
\hline$P_{23}$ & $P_{1}, P_{4}, P_{6}, P_{8}, P_{11}, P_{14}, P_{15}, P_{17}, P_{20}, P_{21}$ \\
\hline$P_{24}$ & $P_{2}, P_{3}, P_{5}, P_{7}, P_{10}, P_{12}, P_{16}, P_{18}, P_{19}, P_{22}$ \\
\hline
\end{tabular}

Table 4 Distance 3 Between Cyclic Permutations

\begin{tabular}{||ll||}
\hline \hline From & To \\
\hline \hline$P_{1}$ & $P_{5}, P_{12}, P_{13}, P_{16}, P_{18}, P_{19}, P_{22}$ \\
$P_{2}$ & $P_{4}, P_{9}, P_{14}, P_{17}, P_{20}, P_{21}, P_{23}$ \\
$P_{3}$ & $P_{6}, P_{8}, P_{9}, P_{11}, P_{17}, P_{20}, P_{21}$ \\
$P_{4}$ & $P_{2}, P_{10}, P_{12}, P_{13}, P_{19}, P_{22}, P_{24}$ \\
$P_{5}$ & $P_{1}, P_{9}, P_{11}, P_{14}, P_{15}, P_{17}, P_{20}$ \\
$P_{6}$ & $P_{3}, P_{7}, P_{10}, P_{12}, P_{13}, P_{18}, P_{22}$ \\
$P_{7}$ & $P_{6}, P_{11}, P_{14}, P_{15}, P_{17}, P_{21}, P_{23}$ \\
$P_{8}$ & $P_{3}, P_{10}, P_{16}, P_{18}, P_{19}, P_{22}, P_{24}$ \\
$P_{9}$ & $P_{2}, P_{3}, P_{5}, P_{12}, P_{18}, P_{22}, P_{24}$ \\
$P_{10}$ & $P_{4}, P_{6}, P_{8}, P_{15}, P_{20}, P_{21}, P_{23}$ \\
$P_{11}$ & $P_{3}, P_{5}, P_{7}, P_{13}, P_{16}, P_{18}, P_{24}$ \\
$P_{12}$ & $P_{1}, P_{4}, P_{6}, P_{9}, P_{15}, P_{17}, P_{21}$ \\
$P_{13}$ & $P_{1}, P_{4}, P_{6}, P_{11}, P_{17}, P_{20}, P_{23}$ \\
$P_{14}$ & $P_{2}, P_{5}, P_{7}, P_{16}, P_{19}, P_{22}, P_{24}$ \\
$P_{15}$ & $P_{5}, P_{7}, P_{10}, P_{12}, P_{18}, P_{19}, P_{24}$ \\
$P_{16}$ & $P_{1}, P_{8}, P_{11}, P_{14}, P_{20}, P_{21}, P_{23}$ \\
$P_{17}$ & $P_{2}, P_{3}, P_{5}, P_{7}, P_{12}, P_{13}, P_{19}$ \\
$P_{18}$ & $P_{1}, P_{6}, P_{8}, P_{9}, P_{11}, P_{15}, P_{23}$ \\
$P_{19}$ & $P_{1}, P_{4}, P_{8}, P_{14}, P_{15}, P_{17}, P_{23}$ \\
$P_{20}$ & $P_{2}, P_{3}, P_{5}, P_{10}, P_{13}, P_{16}, P_{22}$ \\
$P_{21}$ & $P_{2}, P_{3}, P_{7}, P_{10}, P_{12}, P_{16}, P_{24}$ \\
$P_{22}$ & $P_{1}, P_{4}, P_{6}, P_{8}, P_{9}, P_{14}, P_{20}$ \\
$P_{23}$ & $P_{2}, P_{7}, P_{10}, P_{13}, P_{16}, P_{18}, P_{19}$ \\
$P_{24}$ & $P_{4}, P_{8}, P_{9}, P_{11}, P_{14}, P_{15}, P_{21}$ \\
\hline
\end{tabular}

Table 5 Distance 4 Between Cyclic Permutations

\begin{tabular}{||ll||}
\hline \hline From & To \\
\hline \hline$P_{1}$ & $P_{21}$ \\
$P_{2}$ & $P_{18}$ \\
$P_{3}$ & $P_{19}$ \\
$P_{4}$ & $P_{11}$ \\
$P_{5}$ & $P_{10}$ \\
$P_{6}$ & $P_{14}$ \\
$P_{7}$ & $P_{22}$ \\
$P_{8}$ & $P_{17}$ \\
$P_{9}$ & $P_{23}$ \\
$P_{10}$ & $P_{5}$ \\
$P_{11}$ & $P_{4}$ \\
$P_{12}$ & $P_{16}$ \\
$P_{13}$ & $P_{24}$ \\
$P_{14}$ & $P_{6}$ \\
$P_{15}$ & $P_{20}$ \\
$P_{16}$ & $P_{12}$ \\
$P_{17}$ & $P_{8}$ \\
$P_{18}$ & $P_{2}$ \\
$P_{19}$ & $P_{3}$ \\
$P_{21}$ & $P_{1}$ \\
$P_{22}$ & $P_{7}$ \\
$P_{23}$ & $P_{9}$ \\
$P_{24}$ & $P_{13}$ \\
\hline \hline
\end{tabular}



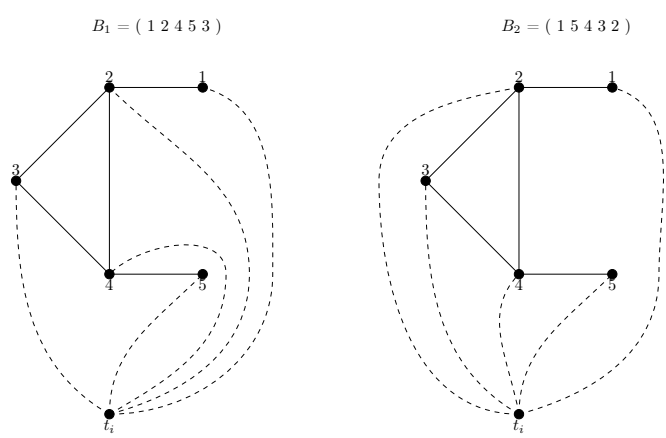

Fig. 3 Graph $G$ and its configurations - type $B$

There is only one drawing of $G$ without crossings shown in Fig. 1. Assume a good drawing $D$ of the graph $G+D_{n}$ in which the edges of $G$ does not cross each other. We will count the number of necessary crossings between two subgraphs $T^{i}$ and $T^{j}$ with $\operatorname{cr}_{D}\left(G, T^{i} \cup T^{j}\right)=0$. In this case, without loss of generality, we can choose the vertex notations of the graph in such a way as shown in Fig. 11. It is easy to see that, in $D$, there are only four different possible configurations of $F^{i}$ summarized in Table 6 see Fig. 2 and 3 . We denote by $\mathscr{M}_{D}$ the set of all configurations that exist in the drawing $D$ belonging to the set $\mathscr{M}$, where $\mathscr{M}=\left\{A_{1}, A_{2}, B_{1}, B_{2}\right\}$.

Table 6 Configurations of graph $G \cup T^{i}$ with vertices denoted of $G$ as in Fig. 1

\begin{tabular}{|l|l|}
\hline$A_{1}:(12543)$ & $A_{2}:(14532)$ \\
\hline$B_{1}:(12453)$ & $B_{2}:(15432)$ \\
\hline
\end{tabular}

Let $X, Y$ be configurations from $\mathscr{M}_{D}$. We shortly denote by $\operatorname{cr}_{D}(X, Y)$ the number of crossings in $D$ between $T^{i}$ and $T^{j}$ for different $T^{i}, T^{j} \in R_{D}$ such that $F^{i}, F^{j}$ have configurations $X, Y$, respectively. Finally, let $\operatorname{cr}(X, Y)=$ $\min \left\{\operatorname{cr}_{D}(X, Y)\right\}$ over all good drawings $D$ of the graph $G+D_{n}$ with $X, Y \in \mathscr{M}_{D}$. The configuration $A_{1}$ is represented by the cyclic permutation $P_{13}=(12543)$ and the configuration $A_{2}$ is represented by the cyclic permutation $P_{22}=(14532)$. As $P_{7}=(12354)$ is the inverse cyclic permutation to the permutation $P_{22}$, then $\operatorname{cr}\left(A_{1}, A_{2}\right) \geq 2$ by Table 3 The similar idea is used for the another cases. Thus, all lower-bounds of numbers of crossings of configurations from $\mathscr{M}$ are summarized in Table 7 .

Table 7 Lower-bounds of numbers of crossings of two configurations from $\mathscr{M}$

\begin{tabular}{c||cccc} 
& $A_{1}$ & $A_{2}$ & $B_{1}$ & $B_{2}$ \\
\hline \hline$A_{1}$ & 4 & 2 & 3 & 3 \\
$A_{2}$ & 2 & 4 & 3 & 3 \\
$B_{1}$ & 3 & 3 & 4 & 2 \\
$B_{2}$ & 3 & 3 & 2 & 4
\end{tabular}

\section{RESULTS}

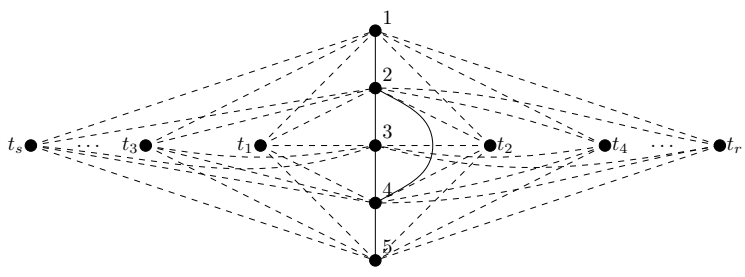

Fig. 4 Good drawing of $G+D_{n}$

Theorem 4.1. Let $G$ be the graph in Fig. 1 and $D_{n}$ is discrete graph with $n$ vertices, then

$\operatorname{cr}\left(G+D_{n}\right)=4\left\lfloor\frac{n}{2}\right\rfloor\left\lfloor\frac{n-1}{2}\right\rfloor+\left\lfloor\frac{n}{2}\right\rfloor$, for $n \geq 1$.

Proof: The theorem is true for $n=1$ and $n=2$. In Fig. 4 there is a drawing of $G+D_{n}$ with $4\left\lfloor\frac{n}{2}\right\rfloor\left\lfloor\frac{n-1}{2}\right\rfloor+\left\lfloor\frac{n}{2}\right\rfloor$ crossings. Thus, $\operatorname{cr}\left(G+D_{n}\right) \leq 4\left\lfloor\frac{n}{2}\right\rfloor\left\lfloor\frac{n-1}{2}\right\rfloor+\left\lfloor\frac{n}{2}\right\rfloor$. We prove the reverse inequality by induction on $n$. For $n \geq 3$, let $D$ be a good drawing of $G+D_{n}$ with less than $4\left\lfloor\frac{n}{2}\right\rfloor\left\lfloor\frac{n-1}{2}\right\rfloor+\left\lfloor\frac{n}{2}\right\rfloor$ crossings. Suppose now that, for $n \geq 3$

$\operatorname{cr}\left(G+D_{n-2}\right) \geq 4\left\lfloor\frac{n-2}{2}\right\rfloor\left\lfloor\frac{n-3}{2}\right\rfloor+\left\lfloor\frac{n-2}{2}\right\rfloor$

and consider such a drawing $D$ of $G+D_{n}$ that

$\operatorname{cr}_{D}\left(G+D_{n}\right)<4\left\lfloor\frac{n}{2}\right\rfloor\left\lfloor\frac{n-1}{2}\right\rfloor+\left\lfloor\frac{n}{2}\right\rfloor$.

The drawing $D$ has the following property:

$\operatorname{cr}_{D}\left(T^{i}, T^{j}\right) \neq 0 \quad$ for all $i, j=1,2, \ldots, n, i \neq j$.

To prove it assume that there are two different subgraphs $T^{i}$ and $T^{j}$ such that $\operatorname{cr}_{D}\left(T^{i}, T^{j}\right)=0$ and let for every integer $s, s<n$, any good drawing of graph $G+D_{s}$ has at least $4\left\lfloor\frac{s}{2}\right\rfloor\left\lfloor\frac{s-1}{2}\right\rfloor+\left\lfloor\frac{s}{2}\right\rfloor$ crossings. Without loss of generality let $\operatorname{cr}_{D}\left(T^{n-1}, T^{n}\right)=0$, one can easy to verify that $\operatorname{cr}_{D}\left(G, T^{n-1} \cup T^{n}\right) \geq 1$. By $\operatorname{cr}\left(K_{5,3}\right)=4$ we give $\operatorname{cr}_{D}\left(T^{k}, T^{n-1} \cup T^{n}\right) \geq 4$ for $k=1,2, \ldots, n-2$. So, for the number of crossings in $D$ we have

$\operatorname{cr}_{D}\left(G+D_{n}\right)=\operatorname{cr}_{D}\left(G \cup \bigcup_{i=1}^{n-2} T^{i}\right)+\operatorname{cr}_{D}\left(T^{n-1} \cup T^{n}\right)+$

$+\operatorname{cr}_{D}\left(G, T^{n-1} \cup T^{n}\right)+\operatorname{cr}_{D}\left(\bigcup_{i=1}^{n-2} T^{i}, T^{n-1} \cup T^{n}\right) \geq$

$\geq 4\left\lfloor\frac{n-2}{2}\right\rfloor\left\lfloor\frac{n-3}{2}\right\rfloor+\left\lfloor\frac{n-2}{2}\right\rfloor+1+4(n-2)=$ $=4\left\lfloor\frac{n}{2}\right\rfloor\left\lfloor\frac{n-1}{2}\right\rfloor+\left\lfloor\frac{n}{2}\right\rfloor$.

This contradicts 1 , and therefore $\operatorname{cr}_{D}\left(T^{i}, T^{j}\right) \neq 0$ for all $i, j=1,2, \ldots, n, i \neq j$. Our assumption on $D$ together with $\operatorname{cr}\left(K_{5, n}\right)=4\left\lfloor\frac{n}{2}\right\rfloor\left\lfloor\frac{n-1}{2}\right\rfloor$ implies that $\operatorname{cr}_{D}(G)+\operatorname{cr}_{D}\left(G, K_{5, n}\right)<\left\lfloor\frac{n}{2}\right\rfloor$.

Thus, we have $r=\left|R_{D}\right|>\left\lfloor\frac{n}{2}\right\rfloor, s=\left|S_{D}\right|<\left\lfloor\frac{n}{2}\right\rfloor$. 
Case 1: $\operatorname{cr}_{D}(G)=0$ as in Fig. 1 .

a) $\left\{A_{1}, A_{2}\right\} \subseteq \mathscr{M}_{D}$ or $\left\{B_{1}, B_{2}\right\} \subseteq \mathscr{M}_{D}$

Without lost of generality if we fix any two $T^{n}$, $T^{n-1} \in R_{D}$ such that $F^{n}, F^{n-1}$ have configurations $A_{1}, A_{2}$, respectively, then $\operatorname{cr}_{D}\left(G \cup T^{n} \cup T^{n-1}, T^{i}\right) \geq 6$ holds by Table 7 for any $T^{i} \in R_{D}$. Using 2, we have

$\operatorname{cr}_{D}\left(G+D_{n}\right)=\operatorname{cr}_{D}\left(K_{5, n-2}\right)+\operatorname{cr}_{D}\left(G \cup T^{n} \cup T^{n-1}\right)+$ $+\mathrm{cr}_{D}\left(K_{5, n-2}, G \cup T^{n} \cup T^{n-1}\right) \geq 4\left\lfloor\frac{n-2}{2}\right\rfloor\left\lfloor\frac{n-3}{2}\right\rfloor+$

$+6(r-2)+3 s+4(n-r-s)+2=$

$=4\left\lfloor\frac{n-2}{2}\right\rfloor\left\lfloor\frac{n-3}{2}\right\rfloor+2 r-s+4 n-10 \geq$

$\geq 4\left\lfloor\frac{n-2}{2}\right\rfloor\left\lfloor\frac{n-3}{2}\right\rfloor+2\left(\left\lfloor\frac{n}{2}\right\rfloor+1\right)+$

$+1-\left\lfloor\frac{n}{2}\right\rfloor+4 n-10 \geq 4\left\lfloor\frac{n}{2}\right\rfloor\left\lfloor\frac{n-1}{2}\right\rfloor+\left\lfloor\frac{n}{2}\right\rfloor$.

b) $\left\{A_{1}, A_{2}\right\} \nsubseteq \mathscr{M}_{D}$ and $\left\{B_{1}, B_{2}\right\} \nsubseteq \mathscr{M}_{D}$.

Without lost of generality if we fix any $T^{n} \in R_{D}$ such that $F^{n}$ has configuration from $\mathscr{M}_{D}$, then $\operatorname{cr}_{D}\left(G \cup T^{n}, T^{i}\right) \geq 3$ holds for any $T^{i} \in R_{D}$. Using the property (2) we have

$$
\begin{aligned}
& \operatorname{cr}_{D}\left(G+D_{n}\right)=\operatorname{cr}_{D}\left(K_{5, n-1}\right)+\operatorname{cr}_{D}\left(G \cup T^{n}\right)+ \\
& +\operatorname{cr}_{D}\left(K_{5, n-1}, G \cup T^{n}\right) \geq 4\left\lfloor\frac{n-1}{2}\right\rfloor\left\lfloor\frac{n-2}{2}\right\rfloor+ \\
& +3(r-1)+2 s+3(n-r-s)= \\
& =4\left\lfloor\frac{n-1}{2}\right\rfloor\left\lfloor\frac{n-2}{2}\right\rfloor+3 n-s-3 \geq \\
& \geq 4\left\lfloor\frac{n-1}{2}\right\rfloor\left\lfloor\frac{n-2}{2}\right\rfloor+3 n+1-\left\lfloor\frac{n}{2}\right\rfloor-3 \geq \\
& \geq 4\left\lfloor\frac{n}{2}\right\rfloor\left\lfloor\frac{n-1}{2}\right\rfloor+\left\lfloor\frac{n}{2}\right\rfloor .
\end{aligned}
$$

In the following three cases we will use the same idea as in the Case $1 \mathrm{~b}$ ). Since $r=\left|R_{D}\right|>\left\lfloor\frac{n}{2}\right\rfloor$, the vertices of degree one noted by 1,5 cannot be separated by 3 -cycle of the graph $G$.

Case 2: $\operatorname{cr}_{D}(G)=1$ as in Fig. 5 .

By a discussion we can easy verify that there is only one type of configuration for $F^{i}$ represented by $P_{5}=(14325)$.

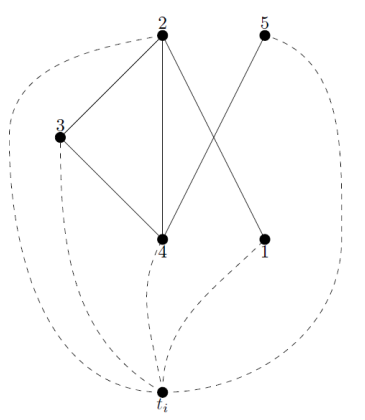

Fig. 5 Good drawing of $G \cup T^{i}$
Case 3: $\operatorname{cr}_{D}(G)=1$ as in Fig. 6 .

By a discussion we can verify that there are only two type of configurations for $F^{i}$ represented by the cyclic permutations $P_{23}=(14352)$ and $P_{3}=(12435) . P_{19}=(15342)$ is the inverse cyclic permutation to the permutation $P_{3}$. Thus, by Table 4 we give lower-bound of number of crossings of these confrgurations equal to three.
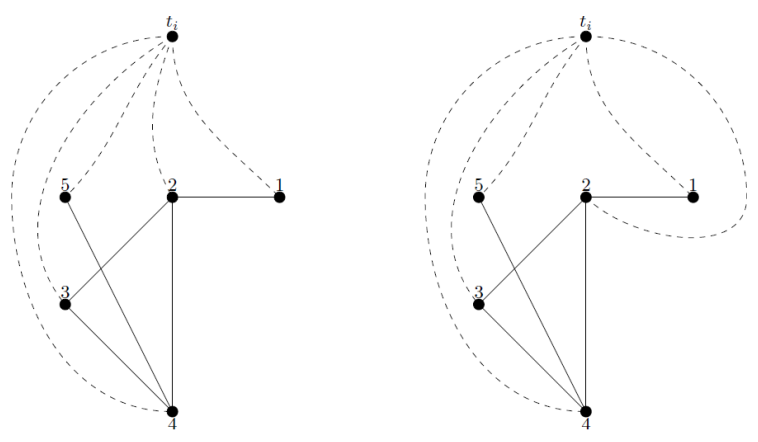

Fig. 6 Good drawing of $G \cup T^{i}$

Case 4: $\operatorname{cr}_{D}(G)=3$ as in Fig. 7

By a discussion we can verify that there is only one type of configuration for $F^{i}$ represented by the cyclic permutation $P_{12}=(13524)$.

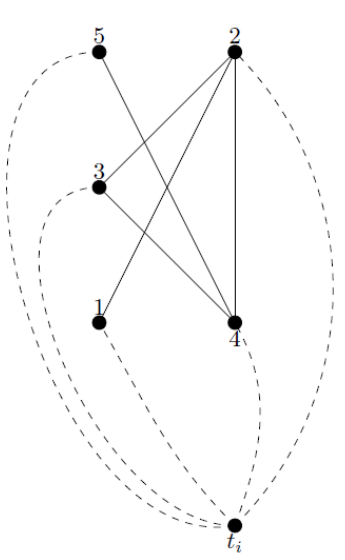

Fig. 7 Good drawing of $G \cup T^{i}$

Theorem 4.2. Let $G$ be the graph in Fig. 1 and $P_{n}$ is a path on $n$ vertices, then

$\operatorname{cr}\left(G+P_{n}\right)=4\left\lfloor\frac{n}{2}\right\rfloor\left\lfloor\frac{n-1}{2}\right\rfloor+\left\lfloor\frac{n}{2}\right\rfloor$ for $n \geq 1$.

We are able to add the edges without crossings in Fig. 4 So the drawing of the graph $G+P_{n}$ with $4\left\lfloor\frac{n}{2}\right\rfloor\left\lfloor\frac{n-1}{2}\right\rfloor+\left\lfloor\frac{n}{2}\right\rfloor$ crossings is obtained. 


\section{CONCLUSIONS}

In this article, we show the proof technique for a crossing number in a given graphs that used the data generated by the software. More significant usage of this software occurs for larger values of $n$ than five. We get 120 cyclical permutations for $n=6,720$ cyclical permutations for $n=7$ which is significantly more than 24 cyclical permutations for $n=5$. For such values, software is an indispensable tool since, we get considerably more complicated graph of distances between cyclic permutations.

\section{ACKNOWLEDGEMENT}

The research was supported by the Slovak VEGA grant No. 1/0389/15. The research was also supported by the internal faculty research project no. FEI-2015-22.

\section{REFERENCES}

[1] LI, B. - WANG, J. - HUANG, Y.: On the crossing number of the join of some 5-vertex graphs and $P_{n}$, International J. Math. Combin., (2008), pp. 70-77.

[2] KLEITMAN, D. J.: The crossing number of $K_{5, n}$, J. Combinatorial Theory, 9 (1970), pp. 315-323.

[3] ZARANKIEWICZ, K.: On a problem of P. Turán concerning graphs, Fund. Math., 41 (1955), pp. 137145.

[4] HERNÁNDEZ-VÉLEZ, C. - MEDINA, C. SALAZAR, G.: The optimal drawing of $K_{5, n}$, Electronic Journal of Combinatorics, 21(4) (2014), 29 pp.

[5] WOODALL, D. R.: Cyclic-order graphs and Zarankiewicz's crossing number conjecture, J. Graph Theory, 17 (1993), pp. 657-671.
Received June 22, 2017, accepted August 9, 2017

\section{BIOGRAPHIES}

Štefan Berežný was born on 1974. In 1998 he graduated (MSc) at the Faculty of Science, P.J. Šafárik University in Košice in the field of discrete mathematics. He defended his $\mathrm{PhD}$ in the field of mathematical optimization in 2005; his thesis title was "Optimization problems on graphs with categorization of edges". Since 1998 he is working as a assistant professor at the Department of Mathematics and Physics on the Air Force Academy of gen. M. R. Štefánik in Košice. His scientific research is focusing on mathematical optimization on graphs, complexity of algorithms, and applied mathematics. In 2004 he went to the Department of Mathematics FEEI TUKE, where he is still a lecturer at the Department of Mathematics and Theoretical Informatics FEEI TUKE. In addition, he also investigates questions related to the use of mathematical software in teaching mathematics courses and appropriate creating of computer laboratories at the department.

Michal Staš was born on 1983. In 2006 he graduated (MSc) at the Faculty of Science, P.J. Šafárik University in Košice in the field of discrete mathematics. He defended his $\mathrm{PhD}$ in the field of discrete mathematics - set theory in 2010; his thesis title was "Hurewicz theorem of analytic sets that are not F-sigma and non-regular sets". Since 2010 he is working as a assistant professor at the Department of Mathematics and Theoretical Informatics FEEI TUKE. His scientific research is focusing on mathematical optimization on graphs, axiom set theory, and applied mathematics. 